\title{
Raphe nuclei echogenicity changes in obsessive-compulsive disorder
}

\author{
Nahid Mohammadzade ${ }^{1}$, Mohammad Reza Shalbafan ${ }^{2}$, Seyed Vahid Shariat ${ }^{2}$, Babak Zamani ${ }^{3}$, \\ Behnam Shariati ${ }^{4}$, Fateme Omrani ${ }^{1}$, Mohammad Rohani*1
}

Received: 12 Jan $2018 \quad$ Published: 24 Sep 2018

\begin{abstract}
Background: Dysregulation of serotonin system is hypothesized to play the main role in the etiology of obsessive-compulsive disorder (OCD). Transcranial sonography (TCS) is a helpful noninvasive and low-cost tool for the assessment of subcortical brain architectures, mainly basal nuclei, cerebellar central structures- and midbrain. In this study, an ultrasound assessment was performed for a sample of the patients with OCD and healthy control group to evaluate echogenicity of midbrain raphe nuclei (RN).

Methods: A total of 35 patients with OCD and 35 healthy controls of similar age and sex entered the study. Semi-structured clinical interview was performed according to the DSM IV-TR criteria to verify OCD. Echogenicity of the midbrain RN was assessed by an experienced neurologist applying TCS. The echogenicity of the 2 groups was compared using chi- square test. SPSS software (version 18 , PASW) was used for statistical analysis and p-value of less than 0.05 was considered significant.

Results: In this study, 15 OCD patients (42.9\%) and 11 (31.4\%) controls showed decreased echogenicity of midbrain RN. Also, the results of the chi-square test showed that the midbrain $\mathrm{RN}$ echogenicity was not significantly lower in patients with OCD compared to the control group $(\mathrm{p}=0.322)$.

Conclusion: Although decreased midbrain RN echogenicity is a characteristic of patients with major depression, it was not shown in OCD patients in this study, which can be explained by the involvement of RN projections rather that RN serotoninergic neurons.
\end{abstract}

Keywords: Transcranial sonography, Obsessive-compulsive disorder, Raphe nuclei, Echogenicity

Copyright $\odot$ Iran University of Medical Sciences

Cite this article as: Mohammadzade N, Shalbafan MR, Shariat SV, Zamani B, Shariati B, Omrani F, Rohani M. Raphe nuclei echogenicity changes in obsessive-compulsive disorder. Med J Islam Repub Iran. 2018 (24 Sep);32:91. https://doi.org/10.14196/mjiri.32.91

\section{Introduction}

Obsessive-compulsive disorder (OCD) is a prevalent psychiatric disorder marked by obsessions (recurring and relentless thoughts) and compulsions (physical or mental actions to decrease anxiety induced by obsessions) (1). This condition affects $1 \%-3 \%$ of the general population worldwide (2-4); however, in an Iranian survey, its prevalence was reported to be $5.1 \%(5)$. OCD is also a disabling psychiatric disease (6), with remission rate of $10 \%$ after standard pharmacological treatment (7).

Corresponding author: Dr Mohammad Rohani, rohani.m@iums.ac.ir

1. Department of Neurology, Hazrat Rasool Hospital, Iran University of Medical Sciences, Tehran, Iran

2. Mental Health Research Center, Department of Psychiatry, School of Medicine, Iran University of Medical Sciences, Tehran, Iran

3. Department of Neurology, Firouzgar Hospital, Iran University of Medical Sciences, Tehran, Iran

4. Department of Psychiatry, Hazrat Rasool Hospital, Iran University of Medical Sciences, Tehran, Iran
Two main theories are at the center of attention for the neurobiological basis of OCD.

According to one theory, the cortico-striatal-thalamiccortical loop plays a considerable role in the pathogenesis of OCD. Activation or inhibition of parts of this loop can mediate compulsive and impulsive features of OCD (8).

Another theory is based on neurotransmitters and multiple neurotransmitters, including serotonin, dopamine, glutamate, and $\mathrm{GABA}$; which are mentioned as the main

$\uparrow$ What is "already known" in this topic:

Dysregulation of serotonin system is hypothesized to play the main role in the etiology of obsessive-compulsive disorder (OCD). Transcranial sonography (TCS) is a helpful noninvasive and low-cost tool for the assessment of subcortical brain architectures, including midbrain raphe nuclei $(\mathrm{RN})$, which consist the main source of serotonin in the brain.

\section{$\rightarrow$ What this article adds:}

Although decreased midbrain RN echogenicity is a characteristic finding in patients with major depression, this was not shown in OCD patients in this study, which can be explained by the involvement of $\mathrm{RN}$ projections rather that $\mathrm{RN}$ serotoninergic neurons. 
players in causing OCD (8).

Serotonin is one of the main monoamine neurotransmitters in the human body and has an important role in several systems, such as gastrointestinal (GI), hematologic, and neuropsychiatric systems. In addition, its dysregulation in central nervous system (CNS) has been considered as plausible and important etiology in psychiatric conditions, particularly depression and OCD. The main cell bodies, which secret this neurotransmitter, are located in raphe nuclei (9). Decreased level of serotonin has been mentioned as the main etiology of OCD. However, recent studies suggest that its role in OCD is more complicated and some serotonin antagonists, such as second generation antipsychotics (10) and ondansetron (11), have been shown as effective choices for adjunctive therapy of OCD.

Recently, inflammatory processes are also proposed as other pathologic mechanisms $(3,4,12)$. Neuroimaging studies have shown increased gray matter in lentiform nuclei and reduced gray matter in bilateral dorsal medial frontal and anterior part of cingulate gyri in these patients (13). A recent study showed reduced raphe nuclei (RN) echogenicity in OCD patients compared to healthy controls on transcranial sonography (TCS) (8).

Transcranial sonography (TCS) is a helpful noninvasive and low-cost tool for the assessment of subcortical brain architectures, mainly basal nuclei, cerebellar central structures, and midbrain. Also, it is a reliable and sensitive diagnostic method in movement disorders, particularly substantia nigra hyperechogenicity, which is a very distinctive finding in Parkinson's disease (14-16). Moreover, serotonergic brainstem RN changes have been reported in major depression and depressed patients with Parkinson's and Huntington's disease (17-20).

In the present study, a thorough sonographic assessment was done for OCD patients. The purpose of this study was to evaluate midbrain RN echogenicity in OCD patients and compare it to healthy controls.

\section{Methods}

Participants

A total of 35 OCD patients selected from the psychiatric outpatient clinic of Haszrat Rasool hospital participated in this study from January 2016 to December 2016. The sample size was calculated according to previous similar studies (8). Diagnosis of OCD was based on the Diagnostic and Statistical Manual of Mental Disorders, fourth edition (DSM-IV TR). Exclusion criteria were other psychiatric disturbances or recent contemporary neurological or other medical diseases, but concurrent major depression and anxiety were not regarded as exclusion criteria.

Most patients were receiving different types of medications, including antidepressants and/or an adjunct new generation or atypical antipsychotic agents. None of the patients received cognitive behavioral therapy during the study period. A total of 35 healthy persons, selected from medical students and hospital staff, were age and sex matched with the case group. After providing full explanation about the study, all participants were given informed consents to sign before their participation. This study was conducted in accordance with the 1975 Declaration of Helsinki. All OCD patients and healthy people underwent TCS by an experienced neurologist.

\section{Clinical assessment}

To quantify the existence of OCD subsymptoms, the Yale-Brown Obsessive-Compulsive Symptom Checklist was used.

\section{Brain parenchyma sonography}

Each participant underwent sonographic evaluation as well as psychiatric assessments on the same day. TCS was done by an experienced neurologist through the temporal bone window using a phased-array ultrasound system (the Osnos 5500 ultrasound system, Sonsite), with a $2.5 \mathrm{MHz}$ probe (S3 probe) and penetration depth of $14-16 \mathrm{~cm}$. The examiner was blinded to the participants. The echogenicity of the $\mathrm{RN}$ was assessed using a semi-quantitative visual

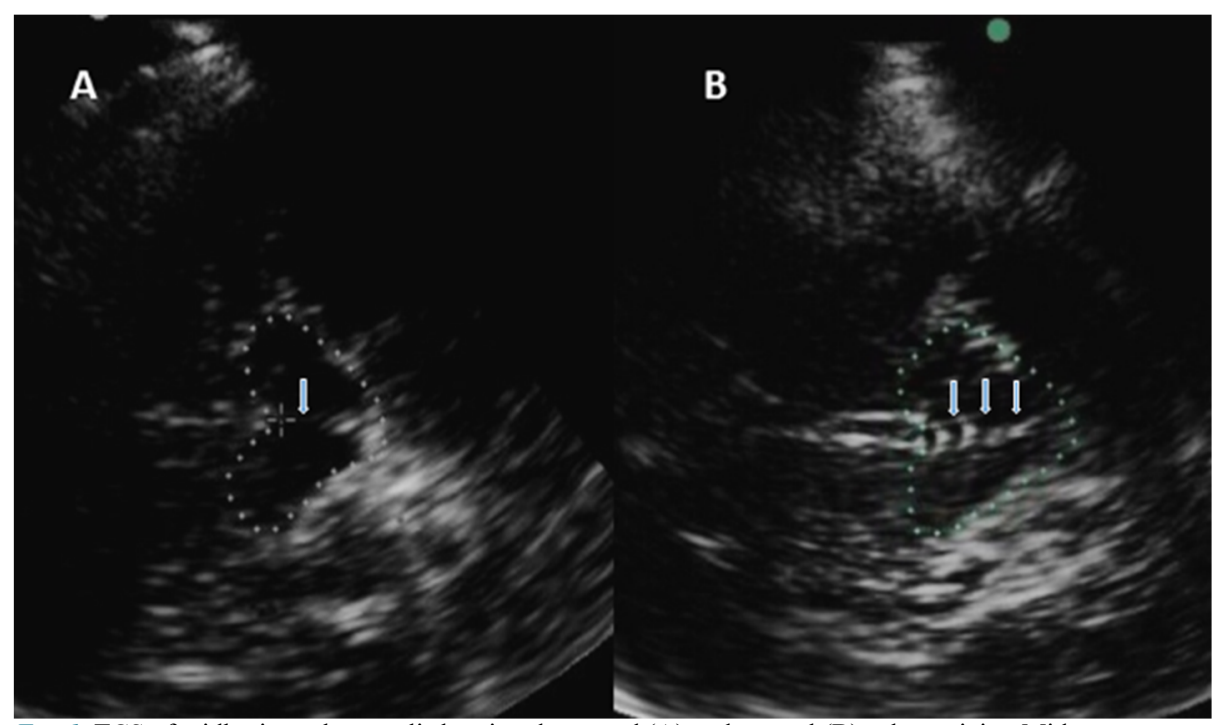

Fig. 1. TCS of midbrain raphe nuceli showing decreased (A) and normal (B) echogenicity. Midbrain's contour is highlighted with dotted lines and arrows points to midline raphe nuclei. 
scale, normal echogenicity with continuous echogenic midline structures, and hypoechogenicity with absent or dotted midline structures (Fig. 1).

\section{Statistical analysis}

To analyze the data, SPSS version 18 (PASW) was used. Mean \pm standard deviation (SD) and frequency percentages were representative of quantitative and qualitative variables, respectively. Chi-square test was performed to compare the frequency of decreased echogenicity among the 2 groups. A p-value of less than 0.05 was considered significant.

\section{Results}

A total of 35 OCD patients and 35 healthy controls entered the study; of them, 27(39\%) were male and 43 $(61 \%)$ were female $(p=0.806)$. The mean age in OCD patients and healthy controls was $36(\mathrm{SD}=12.6)$ and 32 $(\mathrm{SD}=8.2)$ years $(\mathrm{p}=0.141$, age range from 18 to $65 \mathrm{yrs}$.), respectively. In the OCD group, 15 patients showed decreased echogenicity of brainstem RN (42.9\%), whereas 11 persons showed decreased echogenicity in the control group $(31.4 \%)(p=0.322)$ (Table 1$)$.

Another comparison was made in OCD group between patients with and without depression as comorbidity. Also, it was found that 15 out of $35(42.9 \%)$ OCD patients had depression and $7(46.7 \%)$ of them had decreased midbrain RN echogenicity. In OCD patients without depression, 8 patients out of 20 had hypoechoic midbrain RN (40\%). Comparison of the frequency of decreased echogenicity in OCD patients with and without depression revealed no significant differences $(p=0.693)$ (Table 1$)$.

Moreover, 22 OCD patients were receiving medications, mostly SSRIs. There was no prominent difference in raphe echogenicity between these 2 groups $(45.5 \%$ of the patients with and $38.5 \%$ of those without treatment had decreased echogenicity of raphe nuclei, $\mathrm{p}=0.686$ ) (Table 1 ).

\section{Discussion}

In this study, the brainstem RN echogenicity of OCD patients was not significantly lower than the control population. Also, no significant difference was detected in RN echogenicity between OCD patients receiving or not receiving medications and OCD patients with and without depression as comorbidity.

Mavrogiorgou et al. showed hypoechogenicity of brainstem RN in OCD patients, although it was statistically non-significant in comparison to control group. This may be due to altered serotonergic neurons and caudate nuclei hyperechogenicity, reflecting structural or molecular cell alterations (8).

In contrast to this study, they did not compare OCD patients with and without depression and those who received medications with the ones without medications.

Brainstem RN hypoechogenicity is a common and prominent finding in patients suffering from unipolar depression and depressed Parkinsonian and Huntington's patients $(14,18-20,21)$, but this finding has not been reported in multiple sclerosis patients with depression (22).

These findings support the hypothesis that depression in movement disorders is due to involvement of basal limbic system, but in multiple sclerosis it is caused by disorder of basal limbic projections (23). This can be extended to our results as well. Since midbrain RN echogenicity was not significantly different in OCD patients, the main problem in OCD serotoninergic system may remain in $\mathrm{RN}$ projections.

Similar to this finding is signal changes on brain MRI, which have been reported in previous studies, advocating this hypoechogenicity as a structural disruption of mesencephalic raphe structures (24). Tissue echogenicity correlates with tissue impedance, and hypoechogenicity demonstrates tissue microarchitecture changes, which may be explained by alteration of tissue cell density, the composition of interstitial matrix, or transformation of fiber tract integrity (24).

Serotonin deficit has been mentioned in pathophysiology of OCD. Dysfunction of serotonergic system has been suggested based on the effectiveness of SSRIs in the treatment of these patients. In-vivo imaging studies with PET found diminution of cortical 5-HT2A availability in drug-naïve OCD patients compared to controls applying [11C] MDL 100907, a selective 5-HTA2 radio ligand, supporting a relationship between serotonin (5-HT2A) receptors and OCD (25). In contrast to this study, another study (26) did not show a convincing difference in cortical 5-HT2A availability of OCD patients not on medication compared to healthy controls using the same ligand. Interestingly, they found a powerful correlation between orbitofrontal cortex [11C], MDL 100907 binding, and age at disease onset; earlier disease onset was associated with higher binding (27). They did not find differences in cortical HTA2 binding between OCD patients and control group, instead, they found increased 5-HTA2 binding in the nucleus caudalis of OCD patients. Considering the availability of the serotonin transporter, the results are not

\begin{tabular}{|c|c|c|c|c|}
\hline Echogenicity of raphe nuclei & \multicolumn{2}{|c|}{$\begin{array}{c}\text { OCD patients } \\
n(\%)\end{array}$} & $\begin{array}{c}\text { Healthy controls } \\
\mathrm{n}(\%)\end{array}$ & $\mathrm{p}$ \\
\hline Hypoechoic & \multicolumn{2}{|c|}{$15(43 \%)$} & $11(31 \%)$ & 0.322 \\
\hline Normal echogenicity & \multicolumn{2}{|c|}{$20(57 \%)$} & $24(69 \%)$ & \\
\hline & Depressed & Non-depressed & & \\
\hline Hypoechoic & $7(47 \%)$ & $8(40 \%)$ & - & 0.693 \\
\hline Normal echogenicity & $\begin{array}{c}8(53 \%) \\
\text { Receiving SSRIs }\end{array}$ & $\begin{array}{c}12(60 \%) \\
\text { Not receiving SSRIs }\end{array}$ & - & \\
\hline Hypoechoic & $10(45.5 \%)$ & $5(38.5 \%)$ & - & 0.686 \\
\hline Normal echogenicity & $12(55.5 \%)$ & $8(61.5 \%)$ & - & \\
\hline
\end{tabular}


always convincing. Presently, the majority of the in-vivo imaging studies disclose a notable reduction in serotonin transporter (SERT) binding of OCD patients in the midbrain (28).

To increase the accuracy of this study, TCS of all patients was done by one experienced neurologist. The examiner was blinded to the patients' diagnoses, and he did not have any conversation with cases.

As limitations of this study, we should mention the small sample size for comparing raphe echogenicity regarding the duration of the disease, age of onset, and disease severity. Another major limitation was patients' selection, which was done qualitatively rather than quantitatively.

We recommend these points to be considered in future studies with larger sample sizes. Furthermore, the accuracy of the findings depend on the quality of the ultrasound system as well as investigator's qualification (29).

\section{Conclusion}

Serotoninergic system plays an important role in the etiology of OCD. TCS is an easy and non-invasive procedure for the assessment of basal ganglia and brainstem raphe nuclei. As the main source of brain serotonin, midbrain RN was assessed with TCS in OCD patients. In contrast to patients with unipolar depression, our OCD patients did not show meaningful decrement in midbrain $\mathrm{RN}$ echogenicity. This can be explained by involvement of RN projections in OCD patients rather than involvement of RN serotoninergic neurons, which is in contrast to patients with unipolar depression.

\section{Conflict of Interests}

The authors declare that they have no competing interests.

\section{References}

1. American Psychiatric Pub; 2013. Desk Reference to the Diagnostic Criteria from DSM-5R; p. 235.

2. Karno M, Golding JM, Sorenson SB, Burnam MA. The epidemiology of obsessive-compulsive disorder in five US communities. Arch. Gen. Psychiatry. 1988;45(12):1094-9.

3. Shalbafan M, Shariat SV, Alavi K, Zeinoddini A, Salehi M, Askari N et al. Celecoxib as an Adjuvant to Fluvoxamine in Moderate to Severe Obsessive-compulsive Disorder: A Double-blind, Placebo-controlled, Randomized Trial. Pharmacopsychiatry. 2015;48(04/05):136-40.

4. Esalatmanesh S, Zeinoddini A, Rahiminejad F, Sadeghi M, Najarzadegan MR, Shalbafan MR et al. Minocycline combination therapy with fluvoxamine in moderate-to-severe obsessivecompulsive disorder: A placebo-controlled, double-blind, randomized trial. Psychiatry Clin Neurosci. 2016;70(11):517-26.

5. Vandad Sharifi M, Radgoodarzi R. Twelve-month prevalence and correlates of psychiatric disorders in Iran: The Iranian Mental Health Survey 2011. Arch Iran Med. 2015;18(2):76-84.

6. Asnaani A, Alpert E, McLean CP, Simpson HB, Foa EB. The effect of treatment on quality of life and functioning in OCD. Compr Psychiatry. 2017;73:7-14.

7. Brakoulias V. Pharmacotherapy for obsessive-compulsive disorder (OCD): predicting response and moving beyond serotonin re-uptake inhibitors. Expert Opin Pharmacother. 2017;18(1):1-3.

8. Mavrogiorgou P, Meves S, Luksnat S, Norra C, Gold R, Juckel G, et al. Transcranial sonography in obsessive-compulsive disorder. J Psychiatr Res. 2013;47(11):1642-8.

9.Charnay Y, Leger L. Brain serotonergic circuitries. Dialogues in Clin Neurosci. 2010;12(4):471-87.

10.Veale D, Miles S, Smallcombe N, Ghezai H, Goldacre B, Hodsoll J. Atypical antipsychotic augmentation in SSRI treatment refractory obsessive-compulsive disorder: a systematic review and metaanalysis. BMC Psychiatry. 2014 Nov 29;14:317.

11.Kalyani BG, Hegde A, Arumugham SS, Narayanaswamy JC, Math SB, Reddy YCJ. Is Ondansetron Augmentation Effective in Obsessive-Compulsive Disorder? J Clin Psychopharmacol. 2017 Jun;37(3):380-1.

12. Ebrahimi Taj FNS, Ghavidel Darestani S, Shirazi E, Javadinia S. Group A $\beta$-hemolytic Streptococcal Infection in Children and the Resultant Neuro-psychiatric Disorder; a Cross Sectional Study; Tehran, Iran. Basic Clin Neurosci 2015;18(1):1-3.

13. Radua J. Voxel-wise meta-analysis of grey matter changes in obsessive-compulsive disorder. Br J Psychiatry. 2009;195(5):393402.

14. Becker G, Bogdahn U, Reichmann H, Reiners K. Degeneration of substantia nigra in chronic Parkinson's disease visualized by transcranial color-coded real-time sonography. Neurology. 1995;45(1):182-4.

15. Walter U, Probst T, Benecke R, Meyer BU, Dressler D. Brain parenchyma sonography discriminates Parkinson's disease and atypical parkinsonian syndromes. Neurology. 2003;60(1):74-7.

16. Berg D, Walter U. Transcranial sonography in movement disorders. Lancet Neurol. 2008;7(11):1044-55.

17. Becker G, Struck M, Lindner A, Burzer K, Retz W. Reduced echogenicity of brainstem raphe specific to unipolar depression: a transcranial color-coded real-time sonography study. Biol Psychiatry 1995;38(3):180-4.

18. Berg D, Schroder U, Klein R, Tatscher T, Benz P. Echogenicity of substantia nigra: association with increased iron content and marker for susceptibility to nigrostriatal injury. Arch Neurol. 2002;59(6):1999-005.

19. Walter U, Herpertz SC, Benecke R, Hoeppner J. Relationship of brainstem raphe echogenicity and clinical findings in depressive states. Psychiatry Res. 2007;155(1):67-73.

20. Krogias C, Eyding J, Gold R, Norra C, Juckel G, et al Depression in patients with Huntington disease correlates with alterations of the brain stem raphe depicted by transcranial sonography. J Psychiatry Neurosci 2011;36(3):187-94.

21. Ghourchian S, Poorkosary K, Malakouti SK, Rohani M. Raphe nuclei echogenicity changes in major depression. Med J Islam Repub Iran. 2014;28(9).

22. Becker T, Seufert J, Hofmann E, Lange KW, Naumann M, Lindner A et al. Parkinson's disease and depression: Evidence for an alteration of the basal limbic system detected by transcranial sonography. J Neurol Neurosurg Psychiatry 1997;63(5):590-96.

23. Becker G, Struck M, Lindner A, Burzer K, Retz W, Bogdahn U, Beckmann, H. Reduced echogenicity of brainstem raphe specific to unipolar depression: A transcranial color-coded real-time sonography study. Biol Psychiatry. 1995;38(3):180-4.

24. Becker G, Lesch KP, Becker T. Basal limbic system alteration in major depression: a hypothesis supported by transcranial sonography and MRI findings. Int J Neuropsychopharmacol. 2001;4(1):21-31.

25. Perani D, Gorini A, Moresco RM, Henin M, Panzacchi A, Matarrese, $M$ et al. In vivo PET study of $5 \mathrm{HT}(2 \mathrm{~A})$ serotonin and $\mathrm{D}(2)$ dopamine dysfunction in drug-naive obsessive-compulsive disorder. Neuroimage. 2008;42(1):306-14.

26. Simpson HB, Bender J, Xu X, Hackett E, Maher MJ, Abi-Dargham, A. Serotonin $2 \mathrm{~A}$ receptors in obsessive-compulsive disorder: a positron emission tomography study with [11C]MDL 100907. Biol Psychiatry. 2011;70(9):897-904.

27. Adams KH, Pinborg LH, Hasselbalch SG, Svarer C, Holm S, Bolwig TG,. Patients with obsessive-compulsive disorder have increased 5HT2A receptor binding in the caudate nuclei. . Int $J$ neuropsychopharmacol. 2005;8(3):391-401.

28. Nikolaus S, Beu M, Müller HW. Cortical GABA, striatal dopamine and midbrain serotonin as the key players in compulsive and anxiety disorders--results from in vivo imaging studies. Rev Neurosci 2010;21(2):119-39.

29. Bouwmans AE, Mess WH, Kessels A, Weber WE. Specificity and sensitivity of transcranial sonography of the substantia nigra in the diagnosis of Parkinson's disease: prospective cohort study in 196 patients. BMJ Open. 2013;3(4). 